\title{
INTRATHORACIC KIDNEY : A Case Report
}

\author{
Lt Col L SATIJA ${ }^{*}$, Maj V MAURYA ${ }^{+}$, \\ Col HARIQBAL SINGH", Brig G BALARANGAIAH (Retd) ${ }^{* *}$
}

MJAFI 2002; 58 : 173-174

KEY WORDS :Congenital; Ectopic kidney; Intrathoracic kidney.

\section{Introduction}

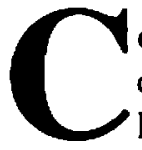

ongenital intrathoracic kidney is a very rare occurrence. Usually, a congenitally ectopic kidney is present in the lower lumbar or pelvic region due to failure to ascend during fetal life. Unlike low ectopic kidneys, which are frequently the site of infection and stone formation, most intrathoracic kidneys function normally and require no medical or surgical therapy once identified. An intrathoracic kidney is mostly discovered as a posterior mediastinal or juxtadiaphragmatic "mass" by chest radiograph. Sonography $[1,4]$ and/or intravenous urography [1-3] allow differentiation from other significant lesions, thus avoiding surgery or biopsy. We discuss here a case of congenital intrathoracic kidney in a child diagnosed by sonography and confirmed by excretory urography.

\section{Case Report}

A 16 month old child who was operated for omphalocele at birth reported for review. This child was detected to have umphalocele on routine antenatal ultrusound. He was delivered by caesar-

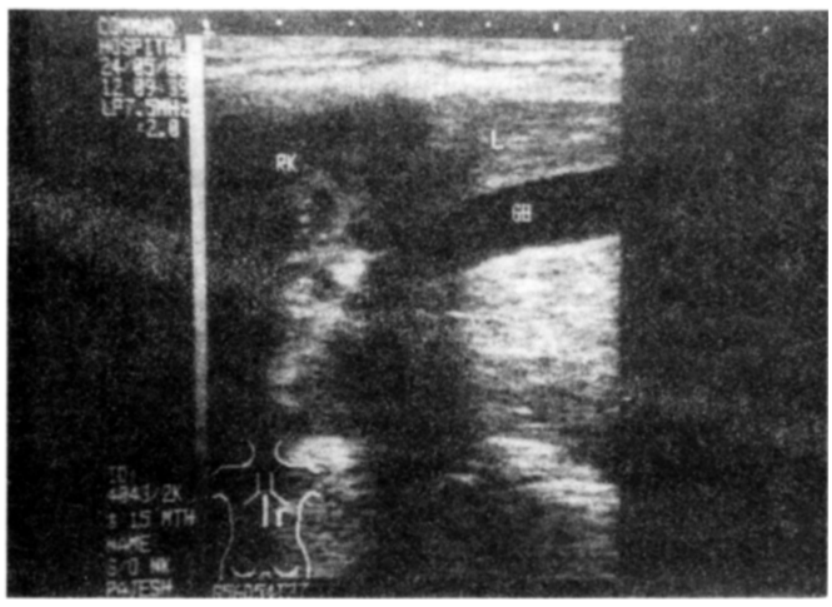

Fig. 1: Ultrasound scan showing suprahepatic right kidney ian section. Anterior abdominal wall repair was carried out electively two days later and again a week later. Presently on physical examination, the child was healthy with normal development for his age. Umbilical hernia was present with expansile cough impulse. Routine chest X-ray films showed a rounded, homogeneous, retrocardiac soft tissue density shadow above the posterior portion of left hemidiaphragm. Ultrasound revealed sub diaphragmatic right kidney (Fig-1) and left kidney in left hemithorax (Fig-2). Intravenous urogram confirmed the sonological findings and demonstrated normal functioning of both kidneys. The right kidney was high up superior to liver and the left kidney was intrathoracic in location (Fig-3 a\&b). Urinanalysis. urine culture, and serum creatinine results were within normal limits. No treatment was necessary.

\section{Discussion}

Unilateral thoracic or superior renal ectopia is a rare anomaly, encountered only in 2 cases in a series of 15,919 autopsies and accounting for less than $5 \%$ of all renal ectopies [1]. Unlike pelvic ectopic kidneys, which sometimes are obstructed, have calculi, or are infected. thoracic ectopic kidneys are usually normal otherwise. They are more common on the left side, like in our case and are usually isolated incidental

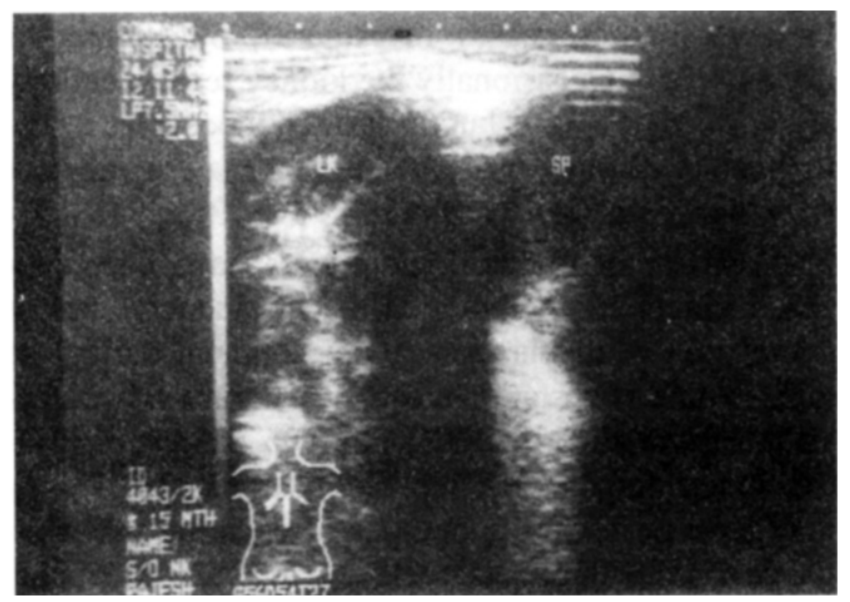

Fig. 2: Ultrisound scan showing supradiaphragmatic left kidney

\footnotetext{
"Classified Specialist (Radiodiagnosis), ${ }^{+}$Trainee (Radiodiagnosis). " Ex-Consultant (Radiodiagnosis), Department of Radiodiagnosis \& Imaging. Command Hospital (Southern Command). Pune - 411040 . "Senior Advisor (Radiodiagnosis), Command Hospital (Westem Command), Chandimandir.
} 

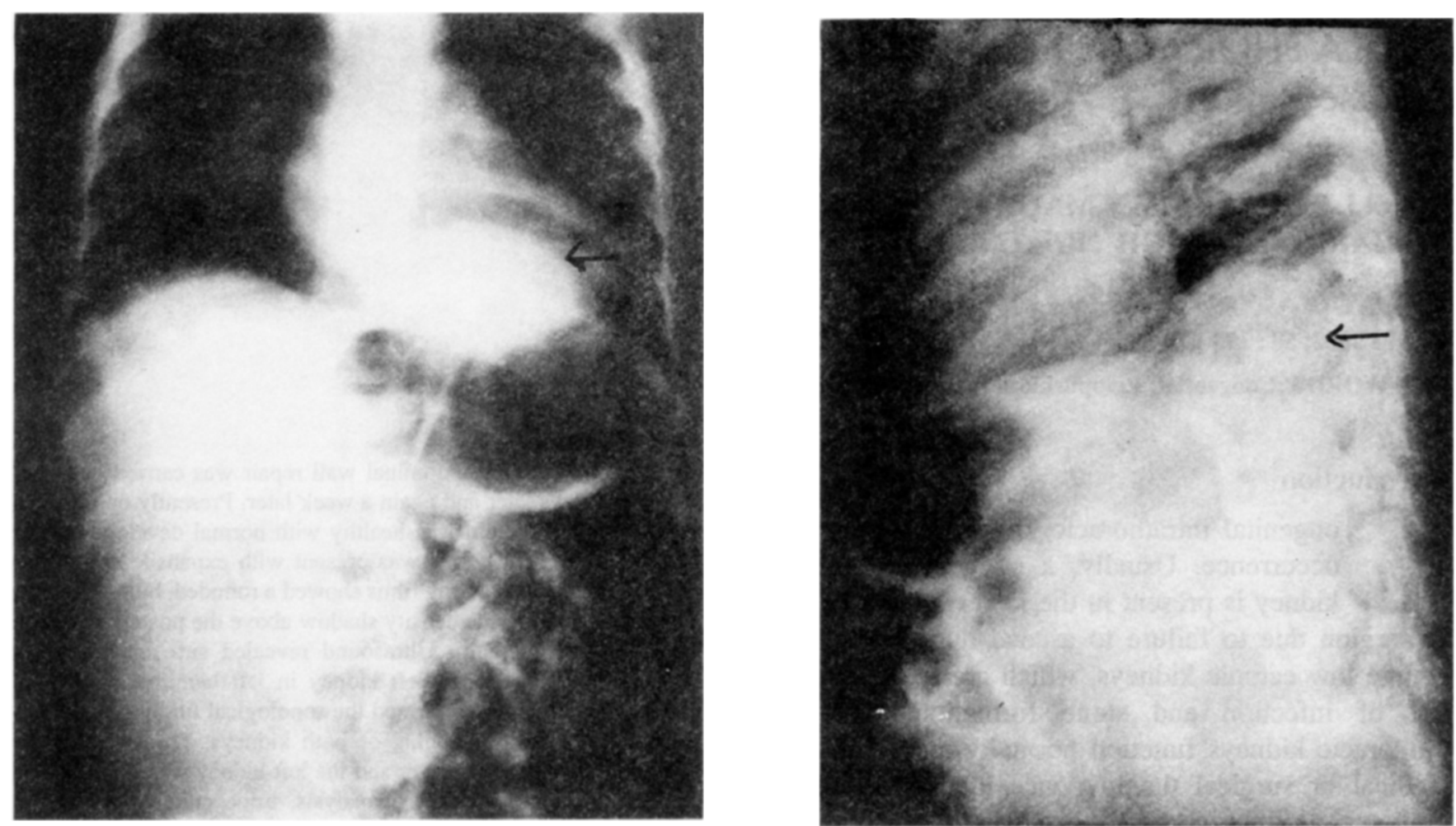

Fig. 3a \& h : Intravenous urogrpahy showing suprahepatic right and intrathoracic left kidney

findings [2]. Three instances of bilateral thoracic kidneys have been reported [3].

Except for the few cases in which thoracic ectopic kidney was thought to be caused by traumatic diaphragmatic distuption [3], most thoracic renal ectopies are assumed (o) be congenital. In some cases, anomalously superior origin of the renal vessels has been found [3], whereas in others the renal vessels are simply longer than normal [3]. The relationship of the superior ectopic kidney to the diaphragm has been variable in those cases investigated by surgery or autopsy [3,5]. Occasionally the kidney resides entirely above the diaphragm, but most often it occupies a posteromedial diaphragmatic defect that may or may not be capped by fibrous tissue [5].

A variety of embryologic causes have been proposed. Superior migration of the metanephros before completion of diaphragmatic development during the eighth week of gestation is the most common hypothesis of these theories [3,5]. In our patient, the intrathoracic left kidney and high placed right kidney may have been caused because of decreased abdominal cavity due to omphalocele and the intra abdominal organs occupying the available space.

In infants and children, the thoracic kidney may resemble a hernia of the foramen of Bochdalek, neurofibroma, or a pericardial cyst [1]. In the adult the thoracic kidney should be differentiated from these and other posterior mediastinal masses and from benign or malignant diaphragmatic, pleural, or pulmonary lesions [1].

This comprehensive review of the literature, accentuates the importance of including ectopic kidney in the differential diagnosis of a diaphragmatic or thoracic mass [1,2]. Improper evaluation could lead to the interpretation of a renal mass to be a neoplasm, resulting in unnecessary thoracotomy. Failure to make an early diagnosis in the past has been due not only to the variable and uncertain clinical manifestations of ectopic kidney but more importantly to the fact that the condition was not considered in differential diagnosis.

\section{References}

1. Moazzendeh AR, Khodadadian P. Potter RT. The intrathoracic kidney. J Thorac Cardiovasc Surg 1977:73:480-2.

2. Donat SM, Donat PE. Intrathoracic kidney : A case report with a review of the world literature. J Urol 1988;140: 131-3.

3. Liddel RM, Rosenbaum DM, Blumhagen JD. Delayed radiologic appearance of bilateral thoracic ectopic kidneys. Amer J Radiol 1989;152:120-2.

4. Kangarloo H. Sukov R. Sample WF, Lipson M, Smith L. Ultrasonic evaluation of juxtadiaphragmatic masses in children. Radiology 1977;125:785-7.

5. N'Guessen G. Stephens FG. Pick J. Congenital superior ectopic (thoracic) kidney. Urology 1984:24:219-28. 